\title{
Nuclear versus Side-Chain Bromination of 4-methoxy toluene by an electrochemical method
}

\author{
K. KULANGIAPPAR and T. RAJU* \\ Electroorganic Division \\ CSIR- Central Electrochemical Research Institute \\ KARAIKUDI - 630 006, INDIA. \\ *Email:traju@cecri.res.in: rajuorganic@yahoo.co.in \\ Telephone no.914565 241558, Fax no.914565 227779
}

\section{Supporting Information}

1. Figure $1{ }^{1} \mathrm{H}$ NMR spectrum for 4- methoxy 3-bromo benzyl bromide

2. Figure $2{ }^{1} \mathrm{H}$ NMR spectrum for 4-methyl 2-bromo acetanilide

3. Figure $3{ }^{13} \mathrm{C}$ NMR spectrum for 4-methyl 2-bromo acetanilide

4. Figure $4{ }^{1} \mathrm{H}$ NMR spectrum for 4-bromomethyl benzoic acid

5. Figure $5{ }^{13} \mathrm{C}$ NMR spectrum for 4 - bromomethyl benzoic acid

6. Figure $6{ }^{1} \mathrm{H}$ NMR spectrum for 3,5-dimethyl 2,6-dibromo anisole

7. Figure $7{ }^{1} \mathrm{H}$ NMR spectrum for 4-benzoyloxy benzyl bromide (crude product) 
4-methoxy 3-bromo benzyl bromide (entry 2), white solid, $59^{\circ} \mathrm{C},{ }^{1} \mathrm{H}$ NMR(400 $\mathrm{MHz}$, $\left.\mathrm{CDCl}_{3}\right) \quad \delta 7.6(\mathrm{~s}, 1 \mathrm{H}), 7.3(\mathrm{~d}, \mathrm{~J}=8.0 \mathrm{~Hz}, 1 \mathrm{H}), 6.8(\mathrm{~d}, \mathrm{~J}=8.0 \mathrm{~Hz}, 1 \mathrm{H}), 4.4(\mathrm{~s}, 1 \mathrm{H})$, 3.9(s, 3H).

4-methyl 2-bromo acetanilide (entry 6), white solild, $114^{\circ} \mathrm{C},{ }^{1} \mathrm{H} \operatorname{NMR}\left(400 \mathrm{MHz}, \mathrm{CDCl}_{3}\right.$ ) $\delta 8.1(\mathrm{~d}, \mathrm{~J}=8.0 \mathrm{~Hz}, 1 \mathrm{H}),(\mathrm{s}, 1 \mathrm{H}), 6.8(\mathrm{~d}, \mathrm{~J}=8.0 \mathrm{~Hz}, 1 \mathrm{H}), 7.3(\mathrm{~s}, 1 \mathrm{H}), 7.1(\mathrm{~d}, \mathrm{~J}=8 \mathrm{~Hz}$, 1H), 2.3(s, 3H), 2.2(s, $\left.\mathrm{CH}_{3}\right) .{ }^{13} \mathrm{C} \mathrm{NMR}\left(400 \mathrm{MHz} \mathrm{CDCl}_{3}\right) \delta$ 168.0, 135.1, 132.7, 132.1. 129.3, 128.6, 121.9, 119.7, 113.3, 77.1, 76.8, 76.5, 24.4, 24.0, 20.5, 20.2.

4-bromomethyl benzoic acid (entry 10), white solid, $224{ }^{\circ} \mathrm{C},{ }^{1} \mathrm{H}$ NMR(400 MHz, DMSO) $\delta 7.9$ (s, 2H), 7.5 (s, 2H), 4.8 ( s, 2H). ${ }^{13} \mathrm{C}$ NMR(400 MHz, DMSO) $\delta$ 167.0, 142.9, 130.7, 129.8, 129.6 90.1, 39.3,33.4.

3,5-dimethyl 2,6-dibromo anisole ( entry 4), white solid, $74^{\circ} \mathrm{C},{ }^{1} \mathrm{H} \mathrm{NMR}\left(400 \mathrm{MHz}, \mathrm{CDCl}_{3}\right)$ $\delta 6.6(\mathrm{~s}, 1 \mathrm{H}), 3.8(\mathrm{~s}, 3 \mathrm{H}), 2.6(\mathrm{~s}, 3 \mathrm{H}) .2 .4(\mathrm{~s}, 3 \mathrm{H})$

4-benzoyloxy benzyl bromide (entry 7), white solid, $108^{\circ} \mathrm{C},{ }^{1} \mathrm{H} \mathrm{NMR}\left(400 \mathrm{MHz}, \mathrm{CDCl}_{3}\right.$ ) $\delta 8.2$ ( d, J=8Hz, 2H) 7.6 (d, J=8Hz, 1H) 7.5 (s, 4H), 7.2 (s, 1H), 7.1 (d, J=8Hz). $4.5(\mathrm{~s}, 2 \mathrm{H})$. 

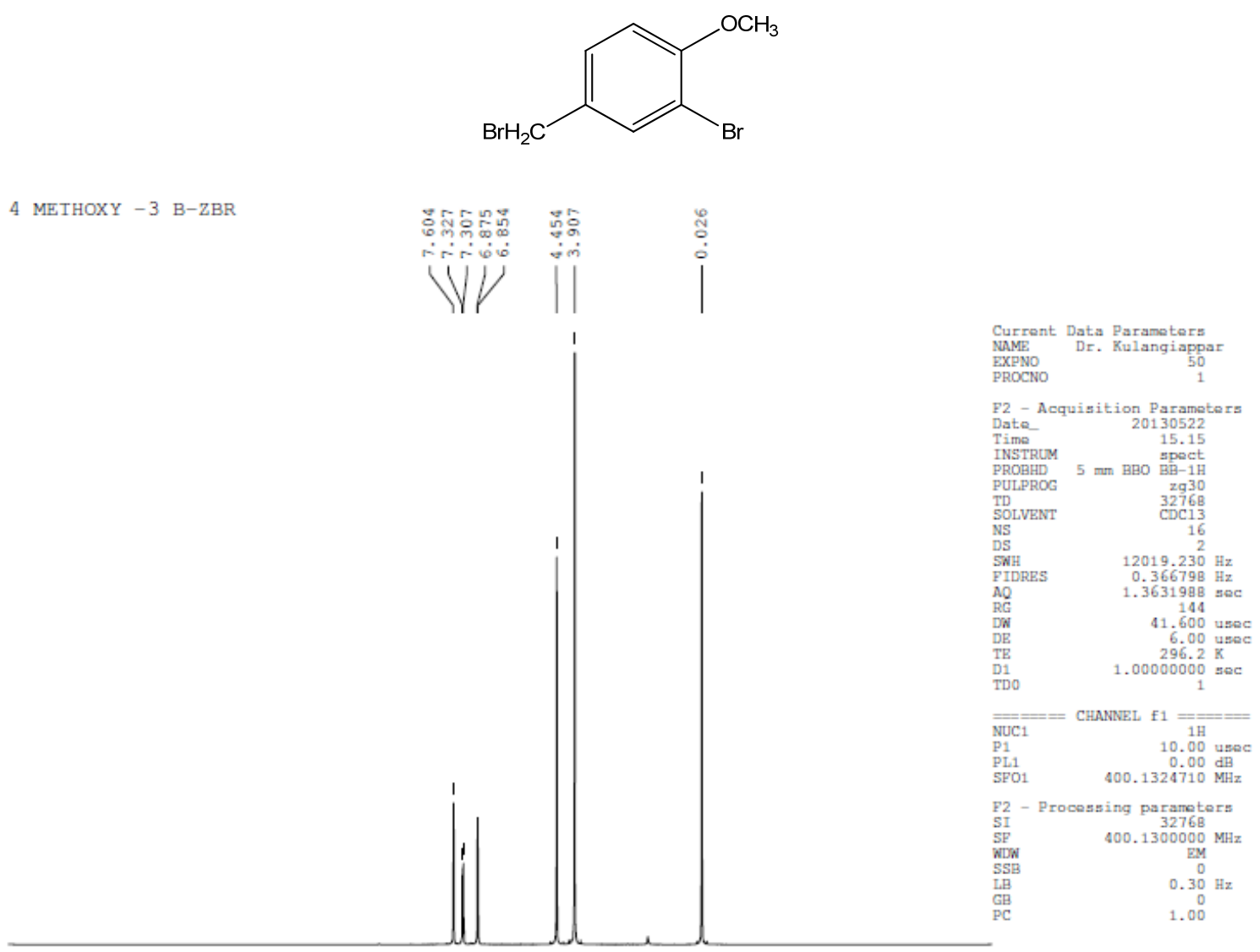

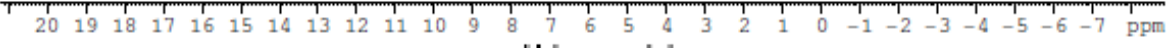

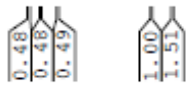

Figure $1{ }^{1}$ HNMR spectrum for 4- methoxy 3-bromo benzyl bromide 
<smiles>CC(=O)Nc1ccc(C)cc1Br</smiles>

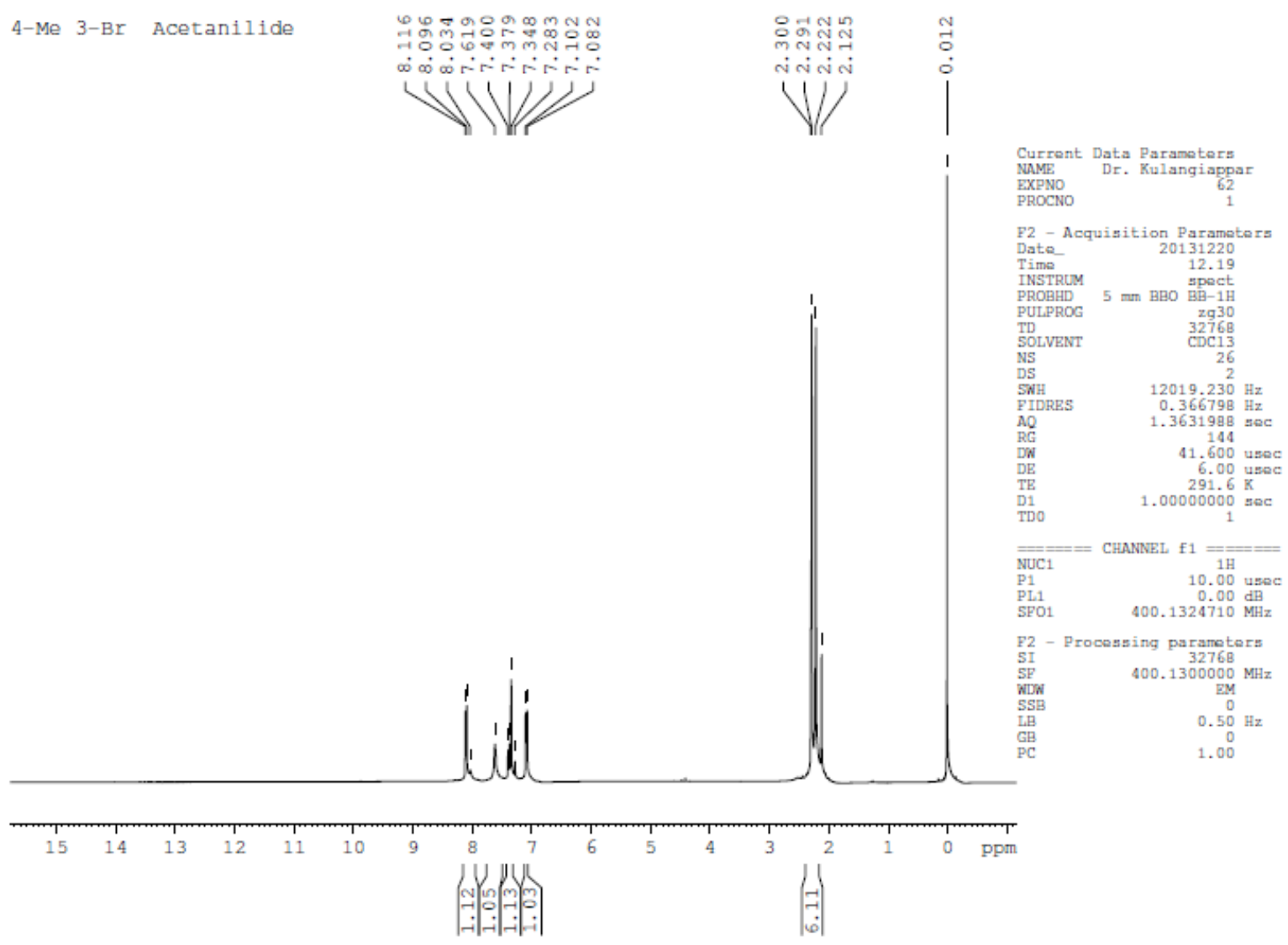

Figure $2{ }^{1}$ HNMR spectrum for 4-methyl 2-bromo acetanilide 
<smiles>CC(=O)Nc1ccc(C)cc1Br</smiles>

4 Me 3 Br acetanilide
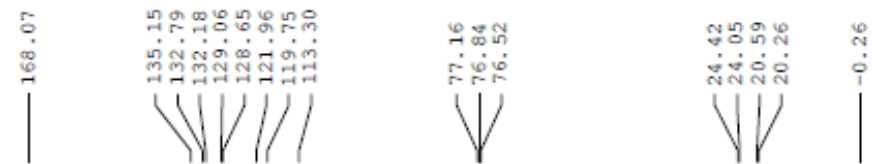

$\begin{array}{lc}\text { Current } & \text { Data Parameters } \\ \text { NAME } & \text { DR. Kulangiappar } \\ \text { EXPNO } & 39 \\ \text { PROCNO } & 1\end{array}$

F2 - Acquisition Parameters

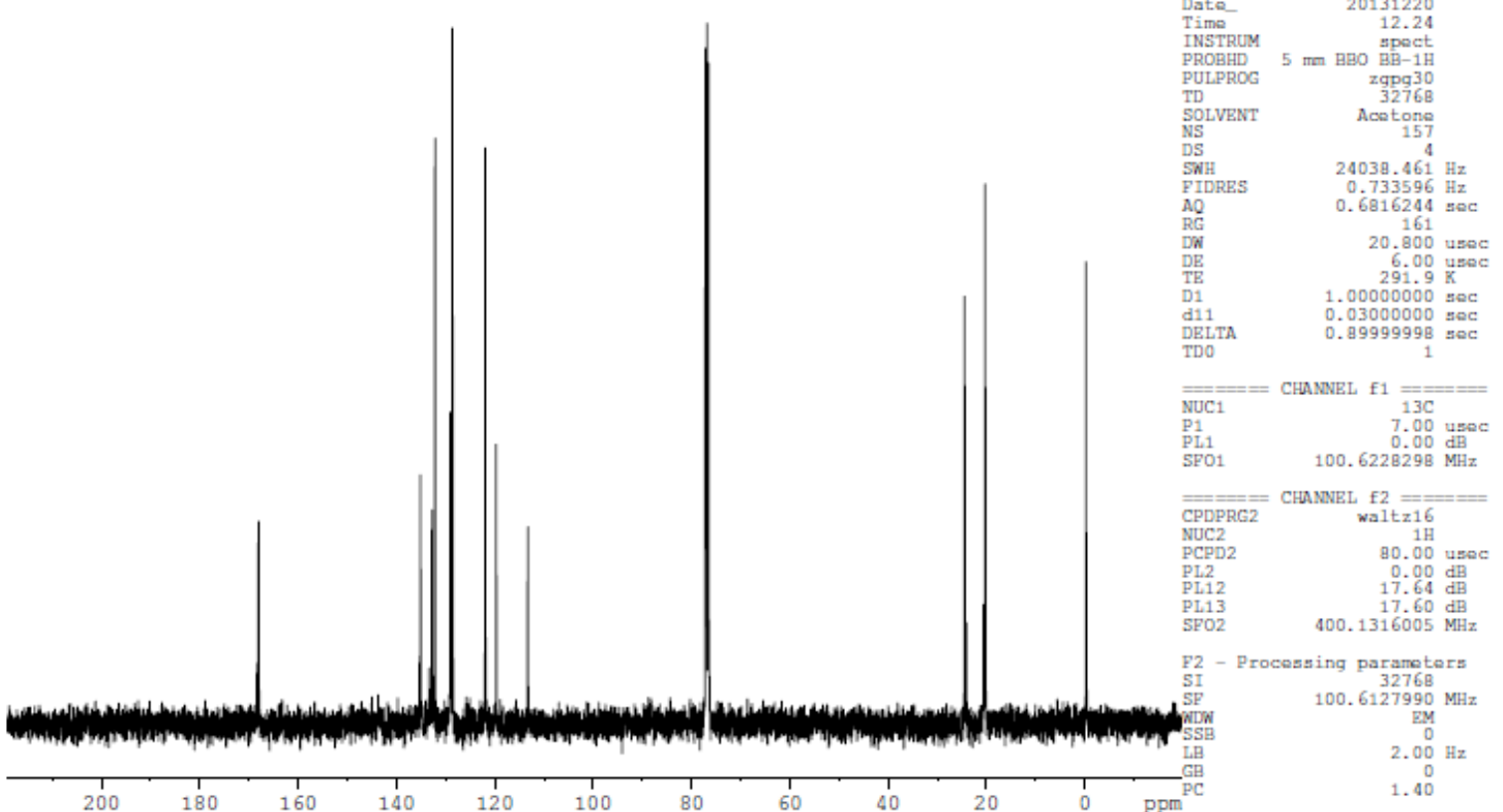

Figure $3{ }^{13} \mathrm{CNMR}$ spectrum for 4-methyl 2-bromo acetanilide 

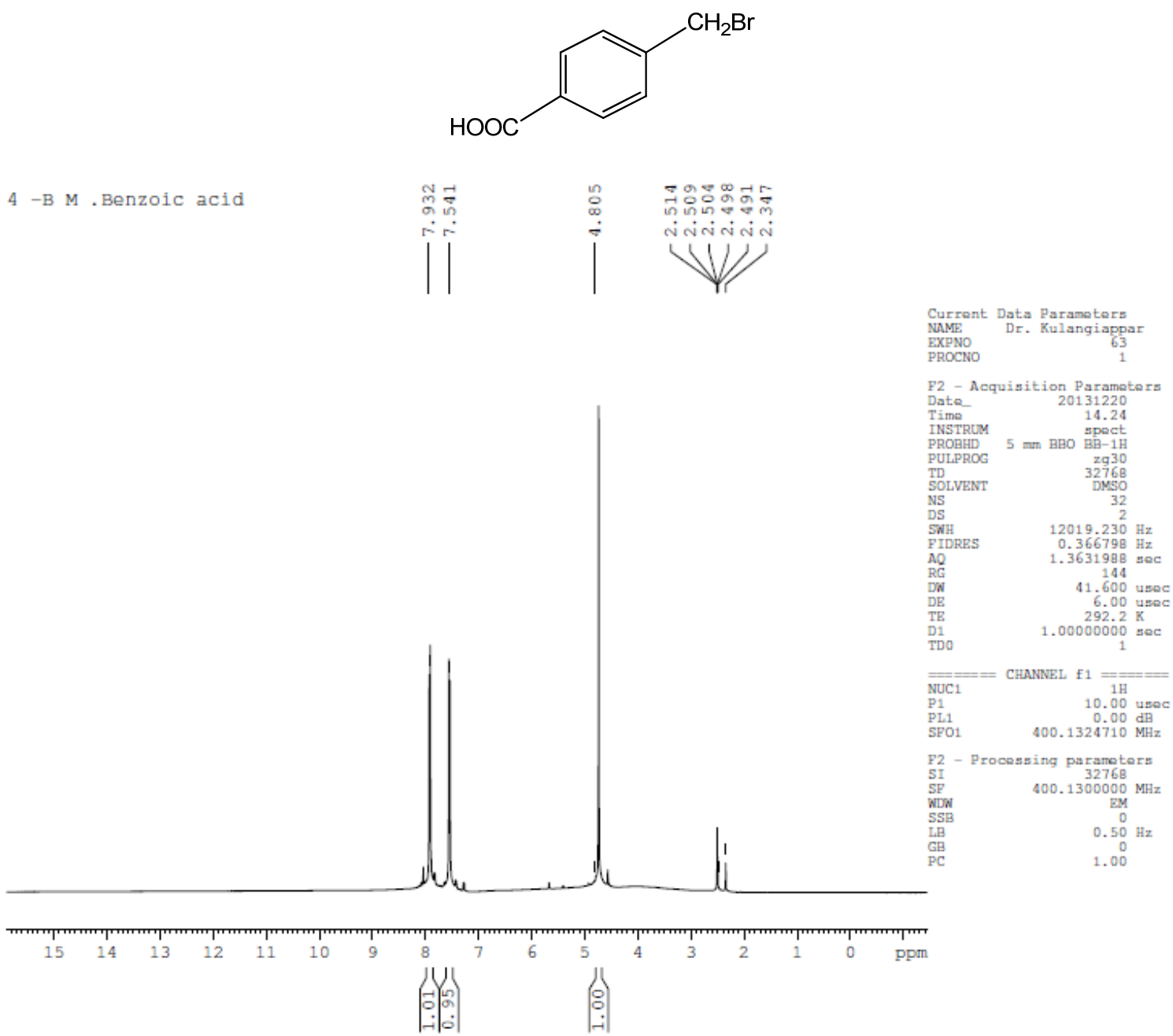

Figure $4{ }^{1}$ HNMR spectrum for 4-bromomethyl benzoic acid 

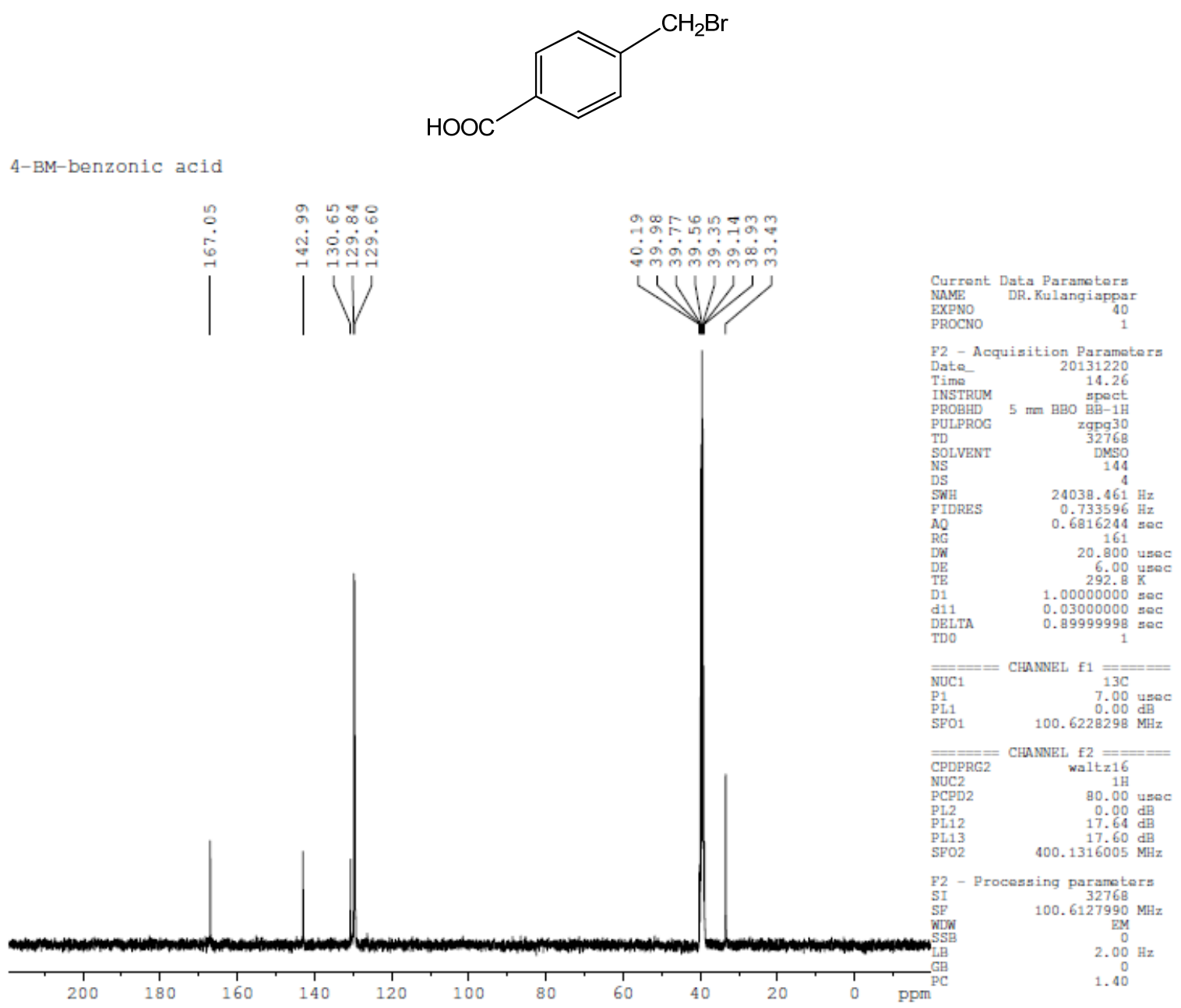

Figure $5{ }^{13} \mathrm{CNMR}$ spectrum for 4 - bromomethyl benzoic acid 

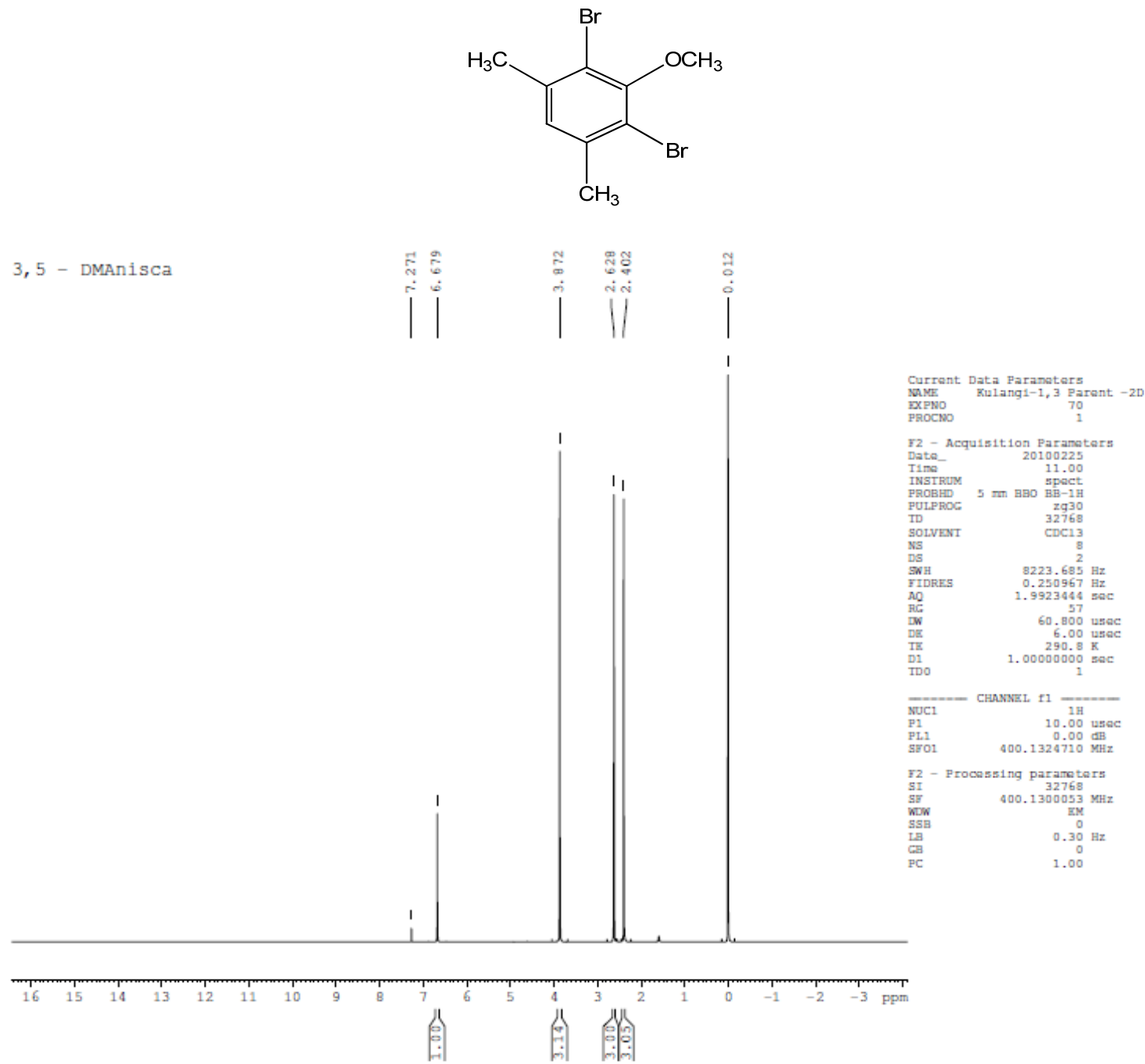

Figure $6{ }^{1} \mathrm{HNMR}$ spectrum for 3,5-dimethyl 2,6-dibromo anisole 


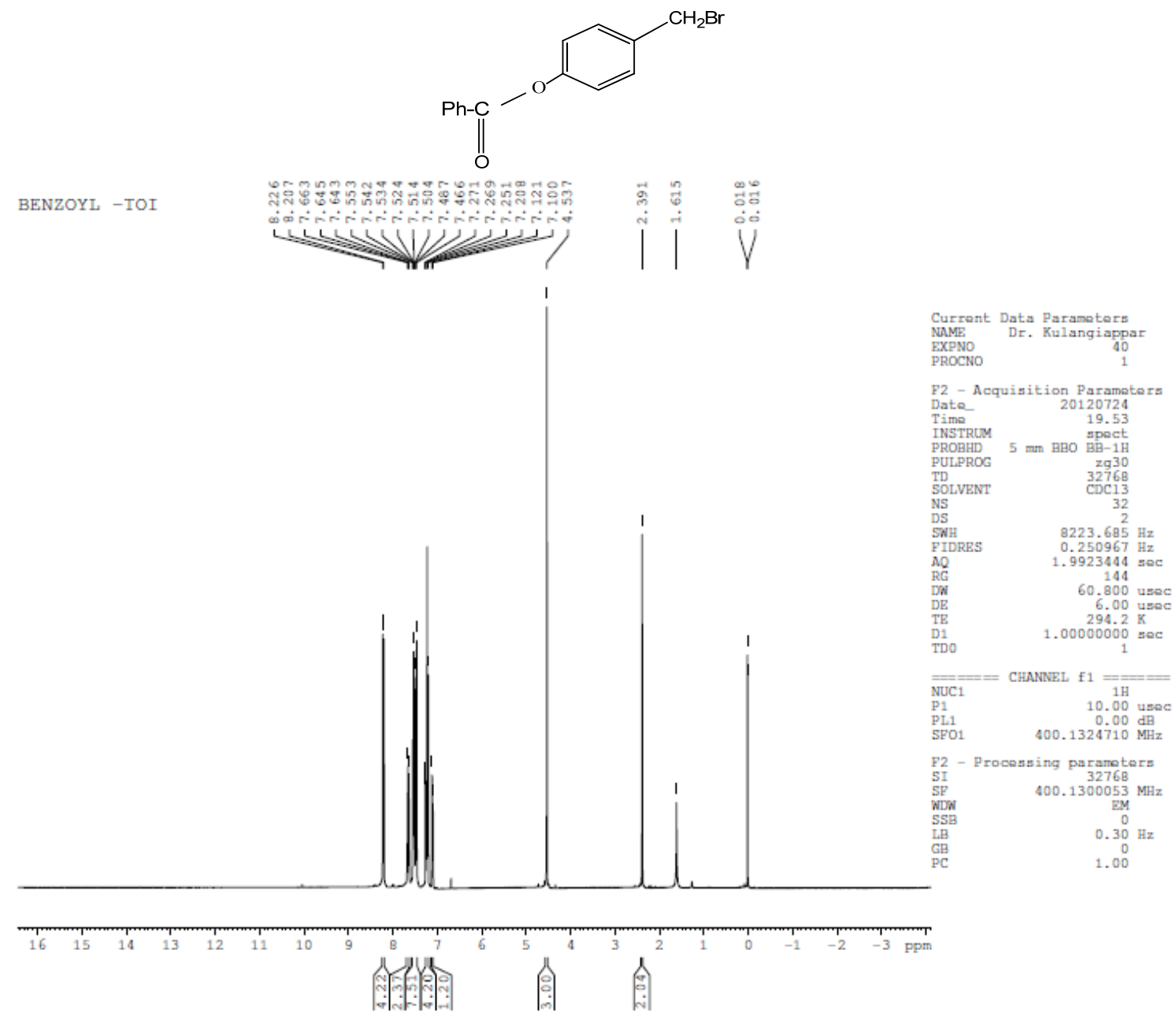

Figure $7{ }^{1} \mathrm{HNMR}$ spectrum for 4-bromomethyl phenyl benzoate (crude product) 